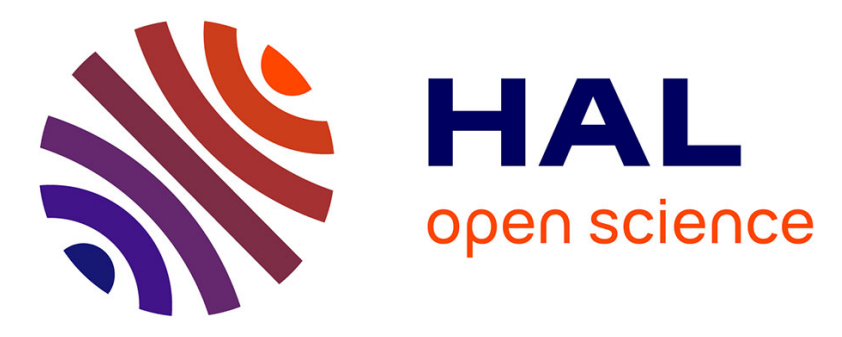

\title{
Hierarchical approach for neonate cerebellum segmentation from MRI: An experimental study
}

Pierre Cettour-Janet, Gilles Valette, Laurent Lucas, Hélène Meunier, Gauthier Loron, Nathalie Bednarek, François Rousseau, Nicolas Passat

\section{- To cite this version:}

Pierre Cettour-Janet, Gilles Valette, Laurent Lucas, Hélène Meunier, Gauthier Loron, et al.. Hierarchical approach for neonate cerebellum segmentation from MRI: An experimental study. International Symposium on Mathematical Morphology (ISMM), 2019, Saarbrücken, Germany. pp.483-495, 10.1007/978-3-030-20867-7_37 . hal-01982960

\section{HAL Id: hal-01982960 \\ https://hal.science/hal-01982960}

Submitted on 31 Mar 2019

HAL is a multi-disciplinary open access archive for the deposit and dissemination of scientific research documents, whether they are published or not. The documents may come from teaching and research institutions in France or abroad, or from public or private research centers.
L'archive ouverte pluridisciplinaire HAL, est destinée au dépôt et à la diffusion de documents scientifiques de niveau recherche, publiés ou non, émanant des établissements d'enseignement et de recherche français ou étrangers, des laboratoires publics ou privés. 


\title{
Hierarchical Approach for Neonate Cerebellum Segmentation From MRI: An Experimental Study ${ }^{\star}$
}

\author{
Pierre Cettour-Janet ${ }^{1}$, Gilles Valette ${ }^{1}$, Laurent Lucas ${ }^{1}$, Hélène Meunier ${ }^{2}$, \\ Gauthier Loron $^{1,2}$, Nathalie Bednarek ${ }^{1,2}$, François Rousseau ${ }^{3}$, Nicolas Passat ${ }^{1}$ \\ 1 Université de Reims Champagne-Ardenne, CReSTIC, France \\ 2 Service de médecine néonatale et réanimation pédiatrique, CHU de Reims, France \\ 3 IMT Atlantique, LaTIM U1101 INSERM, UBL, Brest, France
}

\begin{abstract}
Morphometric analysis of brain structures is of high interest for premature neonates, in particular for defining predictive neurodevelopment biomarkers. This requires beforehand, the correct segmentation of structures of interest from MR images. Such segmentation is however complex, due to the resolution and properties of data. In this context, we investigate the potential of hierarchical image models, and more precisely the binary partition tree, as a way of developing efficient, interactive and user-friendly 3D segmentation methods. In particular, we experiment the relevance of texture features for defining the hierarchy of partitions constituting the final segmentation space. This is one of the first uses of binary partition trees for 3D segmentation of medical images. Experiments are carried out on $19 \mathrm{MR}$ images for cerebellum segmentation purpose.
\end{abstract}

Keywords: 3D segmentation - Binary partition tree - Texture features · MRI · Cerebellum · Premature neonates

\section{Introduction}

Each year, about 15 million babies are born premature, i.e. before 37 weeks of gestation. A part of them will develop cerebral palsy [9] while others will experiment troubles in cognitive performance or behavioral issues [19]. This motivates an active clinical research devoted to predict, at an early stage, the most probable pathologies. In particular, specific information on newborn brain structures can be used as biomarkers. For instance, a correlation between early life cerebellar anatomy and neurodevelopmental disorders was shown recently [32].

In this context, Magnetic Resonance Imaging (MRI) provides an efficient way of observing the newborn brain in a safe fashion. However, MR images of newborns are of lower quality than with adults. Indeed, the used coils are

\footnotetext{
* The research leading to these results has been supported by the ANR MAIA project (http://recherche.imt-atlantique.fr/maia), grant ANR-15-CE23-0009 of the French National Research Agency; and the American Memorial Hospital Foundation.
} 
often not adapted to babies. Moreover, newborns are likely to move during MR acquisitions. As a consequence, specific adaptations are made on standard MR sequences; but they generally result in images with a lower resolution, a decreased signal-to-noise ratio, and possible artifacts. In addition, due to a non-complete maturity of tissues, contrast variations of MR signal and partial volume effects can occur, for instance caused by uncomplete myelination.

An accurate segmentation of brain structures is a prerequisite for carrying out efficiently morphometric measures, further used as biomarkers. While MRI brain structure segmentation in adults has been an active research area for decades, the case of the newborn is more recent. Dedicated (in general, automatic) segmentation methods can be classified into four types [17]: unsupervised [8,21]; parametric [15]; classification [3,18,20,22,36] / atlas fusion [5,12,31]; and deformable models $[34,35]$. These methods aim at segmenting the whole brain, cerebral tissues, or specific structures (e.g., cortex, ventricles, hippocampus), but none of them focused on the cerebellum. Indeed, to our best knowledge, cerebellum segmentation was only considered in adults, in a few papers. In particular, the related approaches were based on texture analysis [28], deformable models $[2,11,16]$, or atlases $[13,26,37]$.

Cerebellum segmentation from neonate MR images is a difficult task, both in an automated and in a manual way. On the one hand, automated segmentation is hardly tractable due to the image properties (low resolution, poor signalto-noise ratio), and the methods initially designed for adult MRI reach their limits in neonate images. On the other hand, manual segmentation remains a complex and error-prone task: it is time-consuming, has to be carried out by an expert, and generally leads to inter- and intra-operator variability. Based on these considerations, we investigate mixed approaches that consist of selecting, in a first, automated step, a reduced subspace of potential segmentation results. In a second, interactive step, the expert-user is then allowed to navigate in this sub-space in order to interactively select and tune a segmentation result.

To reach that goal, we consider hierarchical image models, mainly developed in the field of mathematical morphology, and used for developing connected operators [30]. More precisely, we focus on a specific data-structure, the Binary Partition Tree (BPT, for brief) [29]. The BPT is relevant for two main reasons. First, it enables one to model the image in a multiscale way, from fine anatomical structures to larger classes of tissues. Second, it is built by taking into account not only the intrinsic image information, but also prior knowledge [23] that can be specifically geared towards the selection of structures of interest. As many morphological hierarchies, the BPT is a partially ordered subset of partitions of the image support, organized as a tree structure. It is then possible to easily navigate inside a BPT for interactively defining a cut of the tree, that directly leads to a segmentation result.

In this article, we focus on the first step of our two-step approach, namely the way of building a BPT dedicated to cerebellum segmentation from neonate MR images. Beyond the applicative interest of this work, namely the ability to segment neonates cerebellum, the methodological novelties are manifold. First, we 
consider BPTs for 3D imaging, while they were mainly dedicated to 2D imaging, until now. Second, we propose space / time cost reduction strategies, by coupling BPT construction with a superpixel preprocessing step (Sec. 2.2). Third, we investigate the relevance of texture features for BPT construction (Sec. 2.4). Four, we propose a way of assessing the relevance of a BPT with respect to groundtruth via a Pareto front paradigm (Sec. 3.2). Section 2 describes our methological pipeline. Section 3 provides an experimental discussion. Concluding remarks on perspective works are finally provided in Section 4.

\section{Building a BPT From Neonate MR Images}

In this section, we describe the pipeline that allows us to build a BPT, i.e. a subspace of potential partitions, from the MR image of a neonate, for cerebellum segmentation purpose. We describe the data and the preprocessing steps; we recall the BPT construction algorithm; and we present the chosen features that enable to guide the construction process for finally obtaining a cerebellumoriented BPT.

\subsection{MR Images and preprocessing}

We worked on two MRI datasets: the Neonatal Brain Atlas $\left(\mathrm{ALBERTs}^{4}\right)[6,7]$, and the French Epirmex ${ }^{5}$ dataset; see Fig. 1. ALBERTs data are acquired on a $3 \mathrm{~T}$ Philips Intera scanner. Images have a resolution of $0.82 \times 0.82 \times 1.60$ $\mathrm{mm}$, and dimensions $155 \times 155 \times 189$ voxels, with $\mathrm{TE}=17 \mathrm{~ms}$ and $\mathrm{TR}=4.6$ ms. Epirmex data are T1 MR images acquired in Reims Hospital (Neonatology Service), on a $3 \mathrm{~T}$ Philips MR Imaging DD005 scanner. Images have a resolution of $3 \times 3 \times 3 \mathrm{~mm}$, and dimensions $560 \times 560 \times 90$ voxels, with $\mathrm{TE}=17 \mathrm{~ms}$ and $\mathrm{TR}=1019 \mathrm{~ms}$.

In the sequel, an MR image is noted $I$. It is handled as a function $I: \Omega \rightarrow V$ that associates to each point $x$ of the space $\Omega$ a value $I(x)$ of the set $V$. In our specific case, $\Omega$ is a matrix of voxels, namely a subset of $\mathbb{Z}^{3}$ of the Cartesian grid. The set $V$ is an interval of grey-level values within $\mathbb{N}$.

The MR images are preprocessed. In particular, a denoising by non-local means is performed, based on the BTK library [27]. Then, a bias field correction is applied, based on the N4 algorithm [33]. Finally, in order to reduce the spatial complexity of the BPT construction, an intracranial mask registration is performed, for subdividing the support $\Omega$ of the image $I$ into intra- and extracranial volumes (by abuse of notation, from now on, we will call $\Omega$ the only intracranial area).

${ }^{4}$ (c) Imperial College of Science, Technology and Medicine and I. S. Gousias 2013.

${ }^{5}$ Epirmex is a part of the French epidemiologic study Epipage 2 [1], http://epipage2.inserm.fr. 

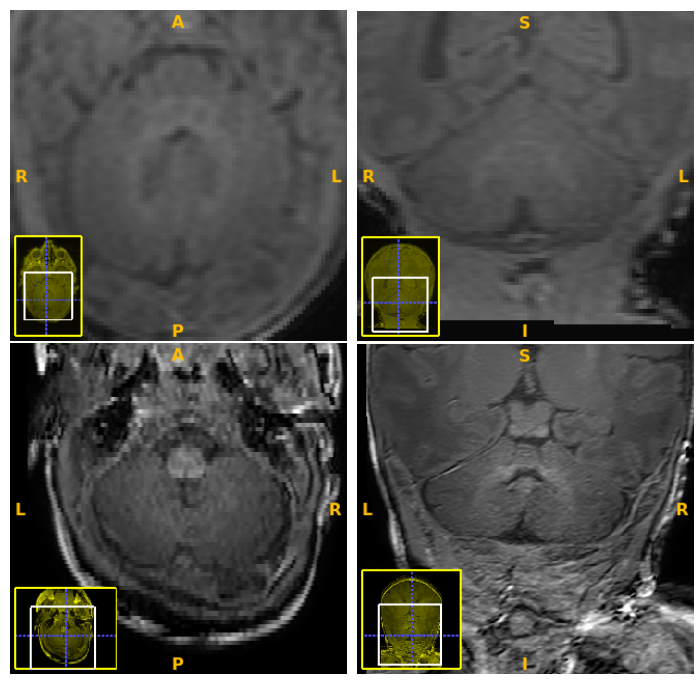

Fig. 1. Examples of MR images (left: axial view; right: coronal view). First row: Epirmex. Second row: ALBERTs.

\subsection{Initial partitioning: Waterpixels}

The BPT models a family of partitions of an initial set (in general, the image support $\Omega$ of $I$ ). The size of the BPT is $\Theta(n)$ where $n$ is the size of this initial set, and the time cost of its construction is $\mathcal{O}(n \log n)$. In practice, these (quasi)linear space / time complexities can lead to high costs, when considering 3D images composed of several millions of voxels.

In order to reduce the space cost of $\Omega$, we investigated superpixel paradigms for simplifying the initial partition induced by $\Omega$ into a reduced partition $\Lambda$. In particular, we considered the waterpixels [14], namely a superpixel approach that applies a seeded watershed on a mixed gradient / distance, saliency map. More precisely, we developed a $3 \mathrm{D}$ version of this $2 \mathrm{D}$ superpixel model, namely the watervoxels [4], in order to allow for a relevant partitioning of an MR image. The main two parameters of waterpixels are the density of seeds (that determines the number of elements of the partition $\Lambda$ ) and the trade-off between the gradient map and the distance map. The experimental setting of these parameters is discussed in Sec. 3.1.

\subsection{BPT construction: Algorithmics}

A BPT is a tree; each of its nodes is a connected region of the image support $\Omega$. A node is either a leaf representing an element of $\Lambda$, or the union of two other neighbouring regions; the root node corresponds to the whole image support $\Omega$.

The BPT construction is a bottom-up process. It proceeds from the leaves, up to the root. This is done by choosing and merging iteratively two adjacent 
regions that minimize a criterion reflecting their similarity. This choice relies on two main notions:

- a region model $M(N)$ which specifies how a region $N$ is characterized (e.g. intensity, texture, geometry); and

- a merging criterion $O\left(N_{1}, N_{2}\right)$ which defines the similarity of neighbouring regions $N_{1}, N_{2}$ and thus the merging order.

A strategy commonly adopted for representing each region is, for instance, to consider their average intensity, and to merge adjacent regions being the most similar.

To model the fact that two points $x$ and $y$ of $\Omega$ are neighbours, we define an adjacency relation on $\Omega$. In practice, in $\mathbb{Z}^{3}$, the standard 6 or 26 -adjacencies defined in digital topology are considered. For the partition $\Lambda$ of $\Omega$, we define an adjacency relation $A_{\Lambda}$ inherited from that of $\Omega$. We say that two distinct sets $N_{1}, N_{2} \in \Lambda$ are adjacent if there exist $x_{1} \in N_{1}$ and $x_{2} \in N_{2}$ such that $x_{1}$ and $x_{2}$ are adjacent in $\Omega$. Then, $G_{\Lambda}=\left(\Lambda, A_{\Lambda}\right)$ is a non-directed graph.

The construction of a BPT is indeed a graph collapsing process. More precisely, the BPT is the data-structure that describes the progressive collapsing of $G_{\Lambda}$ onto the trivial graph $(\{\Omega\}, \emptyset)$. This process consists of defining a sequence $\left(G_{i}=\left(\Gamma_{i}, A_{\Gamma_{i}}\right)\right)_{i=0}^{n}$ (with $\left.n=|\Lambda|-1\right)$ as follows. First, we set $G_{0}=G_{\Lambda}$. Then, for each $i$ from 1 to $n$, we choose the two nodes $N_{i-1}$ and $N^{\prime}{ }_{i-1}$ of $G_{i-1}$ linked by the edge $\left(N_{i-1}, N^{\prime}{ }_{i-1}\right) \in A_{\Gamma_{i-1}}$ that minimizes the chosen merging criterion. We define $G_{i}$ such that $\Gamma_{i}=\left(\Gamma_{i-1} \backslash\left\{N_{i-1}, N^{\prime}{ }_{i-1}\right\}\right) \cup\left\{N_{i-1} \cup N^{\prime}{ }_{i-1}\right\}$; in other words, we replace these two nodes by their union. The adjacency $A_{\Gamma_{i}}$ is defined accordingly from $A_{\Gamma_{i-1}}$. We remove the edge $\left(N_{i-1}, N^{\prime}{ }_{i-1}\right)$, and we replace each edge $\left(N_{i-1}, N^{\prime \prime}{ }_{i-1}\right)$ and / or $\left(N^{\prime}{ }_{i-1}, N^{\prime \prime}{ }_{i-1}\right)$ by an edge $\left(N_{i-1} \cup N^{\prime}{ }_{i-1}, N^{\prime \prime}{ }_{i-1}\right)$. In particular, two former edges may be fused into a single.

The BPT $T$ is the Hasse diagram of the partially ordered set $\left(\bigcup_{i=0}^{n} \Gamma_{i}, \subseteq\right)$. It is built in parallel to the progressive collapsing from $G_{0}$ to $G_{n}$. In particular, $T$ stores the node fusion history. More precisely, we define a sequence $\left(T_{i}\right)_{i=0}^{n}$ as follows. We set $T_{0}=\left(\Gamma_{0}, \emptyset\right)=(\Lambda, \emptyset)$. Then, for each $i$ from 1 to $n$, we build $T_{i}$ from $T_{i-1}$ by adding the new node $N_{i-1} \cup N^{\prime}{ }_{i-1}$, and the two edges $\left(N_{i-1} \cup N^{\prime}{ }_{i-1}, N_{i-1}\right)$ and $\left(N_{i-1} \cup N^{\prime}{ }_{i-1}, N^{\prime}{ }_{i-1}\right)$. The BPT $T$ is then defined as $T_{n}$.

\subsection{Features for BPT construction}

The construction of a BPT requires to choose features involved in the definition of the region model $(M)$, and a merging criterion $(O)$ for assessing the relevance of merging two nodes / regions.

Region model For defining the region model that describes each region / node of the BPT, we first consider the mean grey-level value $(\mu)$. This feature is classically chosen, as it provides a necessary criterion for merging two nodes (i.e. two nodes with very different mean values will not be merged in priority). 
We also consider texture features. Indeed, our hypothesis is that the cerebellum has a specific structure that may be characterized by texture analysis. In particular, we focus on texture indices computed from Haralick co-occurrence matrix [10], that carries second-degree statistics on the image. This co-occurence matrix provides, for each couple of grey-levels of the MR images, the probability of co-occurence between voxels located at a given distance in a chosen direction. In our case, we consider the matrices $\mathcal{M}_{\mathbf{e}_{i}}(i=1,2,3)$ corresponding to co-occurence between neighbouring voxels in the 3 principal orientations, i.e. the 6 -adjacent voxels. More precisely, for any $v, w \in V$ with $V \subset \mathbb{N}$ the interval of MRI values, we have:

$$
\mathcal{M}_{\mathbf{e}_{i}}(v, w)=\frac{1}{|\Omega|}\left|\left\{x \in \Omega \mid I(x)=v \wedge I\left(x+\mathbf{e}_{i}\right)=w\right\}\right|
$$

where $\left\{\mathbf{e}_{1}, \mathbf{e}_{2}, \mathbf{e}_{3}\right\}$ is the canonical orthonormal basis of $\mathbb{Z}^{3}$. From these matrices, we consider the homogeneity $(H)$, contrast $(C)$ and entropy $(E)$ indices, as texture information:

$$
\begin{aligned}
H & =\sum_{i=1}^{3} \sum_{v \in V} \sum_{w \in V} \frac{M_{\mathbf{e}_{i}}(v, w)}{1+|v-w|} \\
C & =\sum_{i=1}^{3} \sum_{v \in V} \sum_{w \in V}|v-w|^{2} \cdot M_{\mathbf{e}_{i}(v, w)} \\
E & =-\sum_{i=1}^{3} \sum_{v \in V} \sum_{w \in V}(v-w) \cdot \log \left(M_{\mathbf{e}_{i}}(v, w)\right)
\end{aligned}
$$

Merging criterion For each pair of adjacent regions $N_{1}, N_{2}$, endowed with one of the four features described above $(\mu, H, C, E)$, we compute the absolute difference of feature values between both regions, noted $O\left(N_{1}, N_{2}\right)$. The lower this value, the more similar the two regions, and the higher the priority for their merging during the BPT construction.

\section{Experiments and Results}

We carried out experiments on ALBERTs (10 MR images) and Epirmex (9 MR images). ALBERTs data are endowed with ground-truth; in particular, the cerebellum region is obtained by union of various labeled subregions. For Epirmex, the $9 \mathrm{MR}$ images were manually segmented by a medical expert in neonatology, for providing cerebellum ground-truth.

\subsection{Watervoxel partition: Parameter setting}

As a preprocessing step to the BPT construction, a simplification of the initial image support $\Omega$ is first carried out. This is done with watervoxels, for subdividing $\Omega$ into a partition $\Lambda$. This partition $\Lambda$ should ideally be composed of regions 
either fully inside or fully outside the cerebellum, in order to avoid initialization errors that the construction of the BPT will not be able to correct (indeed, it merges regions, but never split them).

For a given partition $\Lambda$ of the image support $\Omega$, we define the associated error $\delta$ based on a measure called discordance, introduced in [25]. For the ground-truth segmentation $C \subset \Omega$ of the cerebellum, and the partition $\Lambda \subset 2^{\Omega}$, we define $\delta$ as follows:

$$
\delta=\frac{1}{|C|} \sum_{L \in \Lambda} \min \{|L \backslash C|,|L \cap C|\}
$$

In other words, the discordance measure is defined as the relative quantitative error on the false positives and false negatives with respect to $C$ induced by $\Lambda$, and more precisely by the nodes that partially intersect $G$.

Note that from the very definition of $\delta$, the set $\widehat{S} \subseteq \Lambda$ that best matches $C$ with respect to the discordance measure is such that:

$$
\begin{aligned}
& \forall L \in \Lambda, L \subseteq C \Rightarrow L \in \widehat{S} \\
& \forall L \in \Lambda, L \subseteq \bar{C} \Rightarrow L \notin \widehat{S}
\end{aligned}
$$

In other words, the discordance error depends only on the acceptance or rejection of the $k$ regions $L$ of $\Lambda$ partly meeting $C$. Due to the additive / separable formulation of Eq. (5), the computation of $\delta$ is carried out in linear time $\Theta(k)$, whereas other (similar, although non-equivalent) metrics, e.g. the Dice error, would lead to an exponential $\mathcal{O}\left(2^{k}\right)$ time.

Practically, the partition $\Lambda$ is controlled by two parameters: (1) the density $d \in[0,1]$ of seeds in the image support, that defines the number of regions in the partition as $d .|\Omega|$; and (2) the trade-off parameter $\alpha \in[0,1]$ between the (normalized) distance map $\Delta(\Omega)$ and the (normalized) gradient $\nabla I$ of the MR image, that leads to a map $(1-\alpha) \cdot \nabla I+\alpha \cdot \Delta(\Omega)$. Then, the error defined in Eq. (5) is a 2 -dimensional function $\delta(d, \alpha):[0,1]^{2} \rightarrow \mathbb{R}$. (Note that for $d=1$, we have the trivial partition $\Lambda=\{\{x\} \mid x \in \Omega\}$ and then, $\delta(1,)=$.0 .)

Based on the experimental results summarized in Fig. 2 , we set $d=2,32.10^{-3}$ (resp. $d=1,56 \cdot 10^{-2}$ ) and $\alpha=0,1$ (resp. $\alpha=0,6$ ) for Epirmex (resp. ALBERTs). With these parameters, the mean value of discordance is $\delta=0,311$ (resp. $\delta=0,156)$ for Epirmex (resp. ALBERTs). These values are indeed sufficient for further computing BPTs based on the partition $\Lambda$, with respect to the provided ground-truth of the 9 (resp. 10) MR images. It is however worth mentioning that the discordance error with Epirmex data is much higher than that of ALBERTs. One can then expect worse BPT quality results with clinical images from Epirmex than with ALBERTs images.

\subsection{Segmentation / BPT evaluation}

We then assess the ability of a BPT to provide a correct segmentation. As observed in Sec. 2.3, the nodes of a BPT are regions of the image support $\Omega$. By construction, two nodes $N_{1}$ and $N_{2}$ of the BPT are either disjoint or included: 

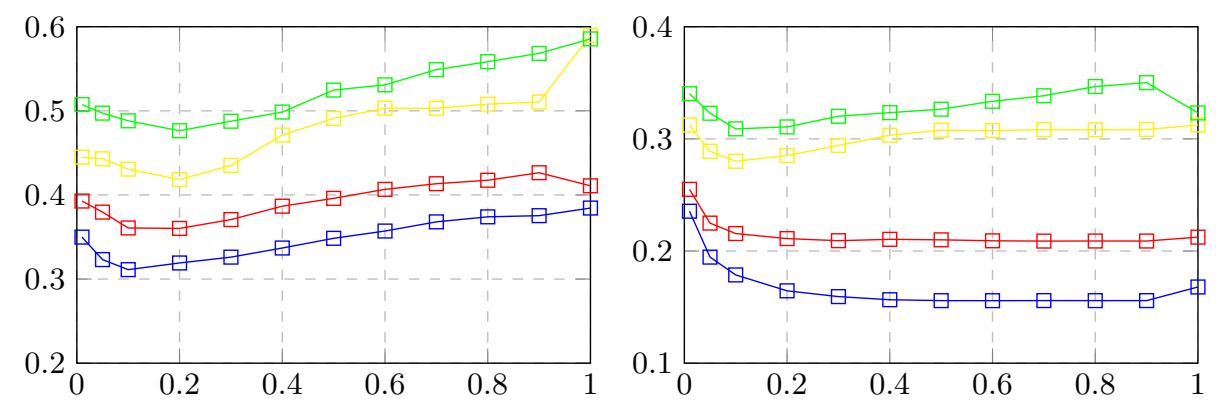

Fig. 2. Discordance $\delta$ (y-axis) depending on the trade-off parameter $\alpha$ between the gradient map and the distance map ( $x$-axis), for various densities $d$. Left: Epirmex dataset. In blue: $d=2,32 \cdot 10^{-3}$ (initial Voronoi cells of $12 \times 12 \times 3$ voxels). In red: $d=1,11.10^{-3}(15 \times 15 \times 4$ voxels $)$. In yellow: $d=5,54 \cdot 10^{-4}(19 \times 19 \times 5$ voxels $)$. In green: $d=3,15 \cdot 10^{-5}(23 \times 23 \times 6$ voxels $)$. Right: ALBERTs dataset. In blue: $d=1,56.10^{-2}$ (initial Voronoi cells of $4 \times 4 \times 4$ voxels). In red: $d=8,00 \cdot 10^{-3}(5 \times 5 \times 5$ voxels $)$. In yellow: $d=2,92.10^{-3}(7 \times 7 \times 7$ voxels $)$. In green: $d=1,95 \cdot 10^{-3}(8 \times 8 \times 8$ voxels $)$.

$N_{1} \cap N_{2} \neq \emptyset \Rightarrow N_{1} \subseteq N_{2} \vee N_{2} \subseteq N_{1}$. A cut of a BPT is a subset $\mathcal{C}=\left\{N_{\star}\right\}$ of pairwise disjoint nodes. Their union $\mathcal{S}=\cup N_{\star} \subseteq \Omega$ provides a segmentation of the image $I$.

The quality of a cut $\mathcal{C} /$ segmentation $\mathcal{S}$ is characterized from two points of view. First, $\mathcal{S}$ has to fit at best the target (here the cerebellum $C$ ), i.e. it should maximize a quality measure [24]. Here, we consider the Dice index, defined as:

$$
D(\mathcal{S}, C)=\frac{2|\mathcal{S} \cap C|}{|\mathcal{S}|+|C|}
$$

Second, $\mathcal{C}$ should contain a minimal set of nodes [25]. Indeed, the less numerous the nodes, the easier the task of defining the cut by automated and/or interactive investigation of the BPT. Then, we consider a second quality measure, namely the size $|\mathcal{C}|$ of the cut.

The best segmentation $\widehat{\mathcal{S}}$, given by the best cut $\widehat{\mathcal{C}}$ of a BPT, should optimize both measures, that is:

$$
\begin{aligned}
& \widehat{\mathcal{S}}=\arg \max D(\mathcal{S}, C) \\
& \widehat{\mathcal{C}}=\arg \min |\mathcal{C}|
\end{aligned}
$$

But, in practice, these are antagonistic.

Thus, in order to assess the relevance of a given BPT, we compute the Pareto front for Eqs. (9-10), that is the set of optimal elements for the couple $(D(\mathcal{S}, C),|\mathcal{C}|)$ in the space $[0,1] \times \mathbb{N}$, for the order relation $\left(\geq_{[0,1]}, \leq_{\mathbb{N}}\right)$.

For each cut $\mathcal{C}$ of the BPT, associated to a segmentation $\mathcal{S}$ of $\Omega$, three information are useful: $a=|\mathcal{S} \cap C|, b=|\mathcal{S}|$, and $c=|\mathcal{C}|$. From this triple $(a, b, c)$, one can recover the Dice and cardinality information of the cut, as $\frac{2 . a}{b+|C|}$ and $c$. 
A cut can be (1) empty; (2) equal to a singleton set corresponding to the root of the BPT; or (3) formed by the union of two cuts, i.e. one in each subtree of the BPT. Based on these facts, the Pareto front can then be built in a recursive, bottom-up fashion as follows.

Let $N$ be a node of the BPT, and $N_{1}, N_{2}$ its children nodes (if $N$ is not a leaf). If $N$ is a leaf, only two cuts can be built from the associated subtree, corresponding to $\{N\}$ and $\emptyset$. They correspond to the triples $(|N \cap C|,|N|, 1)$ and $(0,0,0)$, respectively, that lead to the points of coordinates $(2|N \cap C| /(|N|+$ $|C|), 1)$ and $(0,0)$ in the Pareto space, respectively. These two triples are stored in a set $\mathcal{T}_{N}$. If $N$ is not a leaf, for any triple $\left(a_{1}, b_{1}, c_{1}\right)$ of $\mathcal{T}_{N_{1}}$ and any $\left(a_{2}, b_{2}, c_{2}\right)$ of $\mathcal{T}_{N_{2}}$, one can build a new triple $(a, b, c)=\left(a_{1}+a_{2}, b_{1}+b_{2}, c_{1}+c_{2}\right)$ that corresponds to a unique cut of the (sub-)BPT of root $N$. This includes in particular the empty cut. A supplementary cut, namely $\{N\}$, is not formed by union of two cuts. It corresponds to the triplet $(a, b, c)=\left(a_{1}+a_{2}, b_{1}+b_{2}, 1\right)$ where $\left(a_{1}, b_{1}, c_{1}\right)$ (resp. $\left.\left(a_{2}, b_{2}, c_{2}\right)\right)$ is the triple of maximal value $c_{1}$ (resp. $\left.c_{2}\right)$ in $\mathcal{T}_{N_{1}}$ (resp. $\mathcal{T}_{N_{2}}$ ).

Following this divide-and-conquer algorithmic process, one may build all the points of the Pareto space. However, each computation at a node $N$ creates new triples by Cartesian product between those of the two children nodes. This leads to an exponential space and time complexity, that makes an exhaustive computation practically untractable.

To tackle this issue, three remarks can be made. First, it is not required to process the whole BPT. Indeed, the nodes fully outside $C$ and those with a parent node fully inside $C$ are useless for computing the Pareto front. Then, one can omit them when scanning the BPT, and thus process an ad hoc subtree of the BPT, with a limited subset of leaves.

Second, the computation of the Pareto front for high values of cardinality $|\mathcal{C}|$ is not practically relevant. Indeed, the quality of a BPT lies in its ability to allow for the computation of cuts leading to correct segmentations. However, such cuts should have a reasonably low cardinality for making their selection tractable, both for a human user or an optimal cut procedure. As a consequence, at each stage of the computation, all the triples $(a, b, c)$ with a value $c>t$ for a given threshold value $t$ corresponding to the maximally allowed cardinality may be removed. Note that this optimization requires that each node $N$ stores the two values $|N \cap C|$ and $|N|$, in addition to its set of triples, for allowing the computation of the singleton cut $\{N\}$. This information can be computed in an additive bottom-up fashion, as for the triples $(a, b, c)$.

Third, even with such optimizations, a combinatorial explosion may occur. As a consequence, it is better to compute a (limited but relevant) subset of the points in the Pareto space by only preserving, for each node, a list of $t+1$ triples that lead to the best Dice scores, i.e. one triple for each of the $k \in \llbracket 0, t \rrbracket$ distinct size of cuts $|\mathcal{C}|$. Doing so, the size of the list of triples is bounded by $t+1$ at each node, and the time cost of generation of such list is also bounded by a constant value $(t+1)^{2}$. The obtained Pareto front is not guaranteed to be optimal, but the way to choose the preserved triples allows us to expect a near-to-optimal approximated result. 

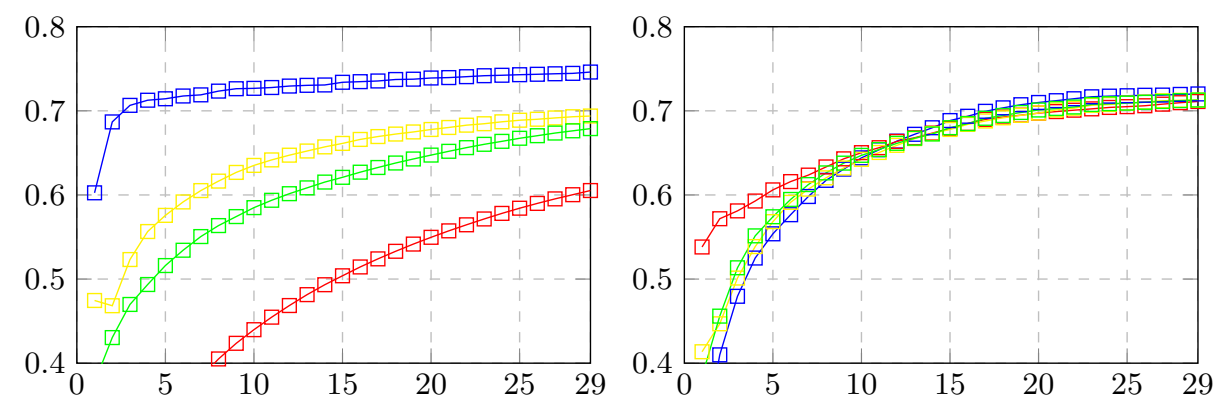

Fig. 3. Pareto front (Dice $D$ in $y$-coordinate vs. size of the cut $|\mathcal{C}|$ in $x$-coordinate) for the BPTs constructed with the four texture features $\mu$ (in blue), $H$ (in red), $C$ (in yellow) and $E$ (in green). The results are obtained by averaging the Dice values over the datasets Left: Epirmex dataset. Right: ALBERTs dataset.

We investigated the BPTs built for each of the four textures $\mu, H, C, E$ (Sec. 2.4). The average results are depicted in Fig. 3. We restricted our study to cuts of maximal cardinality $t=30$.

\section{Conclusion and Perspective Works}

The analysis of the Pareto fronts allows us to observe that the resolution of the MR images has a strong influence on the relevance of using texture features for BPT construction. In particular, for Epirmex data, due to a low resolution, texture features are not able to guide the BPT construction process better than the standard mean feature $(\mu)$. In the case of ALBERTs, i.e. with MR images of higher resolution, one can observe that Homogeneity $(H)$ provides better results than the standard mean feature $(\mu)$ up to cuts of 10 nodes. For cuts of higher cardinalities, the behaviour of the four features are quantitatively comparable.

This tends to prove that (well-chosen) texture features are indeed relevant for carrying out the segmentation of the cerebellum based on BPT paradigms. This robustness remains, however, to be confirmed. Indeed, on the one hand, the current Pareto fronts are built in a near-to-optimal, but non-exhaustive, fashion. On the other hand, the obtained Dice values for a cut of the BPT remain lower than expected for a fully satisfactory segmented cerebellum.

This emphasizes two perspective works. First, we will investigate BPTs built from several features, instead of one. For that purpose, we may rely on recent works on multi-feature BPT [23]. This will allow us to couple texture features, but also to mix them with prior knowledge modeled by atlases. On the other hand, we will experiment various paradigms of gradient, in order to improve the ability of the watervoxel preprocessing to provide initial partitions with greater voxel sizes. As a side effect, we may be able to produce BPT cuts with better Dice scores for a same cardinality. 
Further works will also consist of developing visualization / interaction paradigms to allow a medical expert to easily navigate within a BPT, and observe / define in real time the segmentation associated to a given cut in 3D MR images.

\section{References}

1. Ancel, P.Y., Goffinet, F., EPIPAGE 2 Writing Group: EPIPAGE 2: A preterm birth cohort in France in 2011. BMC Pediatrics 14, 97 (2014)

2. Bogovic, J.A., Bazin, P.L., Ying, S.H., Prince, J.L.: Automated segmentation of the cerebellar lobules using boundary specific classification and evolution. In: IPMI, Proceedings. pp. 62-73 (2013)

3. Cardoso, M.J., Melbourne, A., Kendall, G.S., Modat, M., Robertson, N.J., Marlow, N., Ourselin, S.: AdaPT: An adaptive preterm segmentation algorithm for neonatal brain MRI. NeuroImage 65, 97-108 (2013)

4. Cettour-Janet, P., Cazorla, C., Machairas, V., Delannoy, Q., Bednarek, N., Rousseau, F., Decencière, E., Passat, N.: Watervoxels. HAL Research Report hal02004228 (2019)

5. Coupé, P., Manjón, J.V., Fonov, V., Pruessner, J., Robles, M., Collins, D.L.: Patchbased segmentation using expert priors: Application to hippocampus and ventricle segmentation. NeuroImage 54, 940-954 (2011)

6. Gousias, I.S., Edwards, A.D., Rutherford, M.A., Counsell, S.J., Hajnal, J.V., Rueckert, D., Hammers, A.: Magnetic resonance imaging of the newborn brain: Manual segmentation of labelled atlases in term-born and preterm infants. NeuroImage 62, 1499-1509 (2012)

7. Gousias, I.S., Hammers, A., Counsell, S.J., Srinivasan, L., Rutherford, M.A., Heckemann, R.A., Hajnal, J.V., Rueckert, D., Edwards, A.D.: Magnetic resonance imaging of the newborn brain: Automatic segmentation of brain images into 50 anatomical regions. PLoS One 8, e5999 (2013)

8. Gui, L., Lisowski, R., Faundez, T., Hüppi, P., Lazeyras, F., Kocher, M.: Morphology-based segmentation of newborn brain MR images. In: MICCAI NeoBrainS12, Proceedings. pp. 1-8 (2012)

9. Hack, M., Fanaroff, A.A.: Outcomes of children of extremely low birthweight and gestational age in the 1990s. Seminars in Neonatology 5, 89-106 (2000)

10. Haralick, R.M., Shanmugam, K., Dinstein, I.: Textural features for image classification. IEEE Transactions on Systems, Man, and Cybernetics SMC-3, 610-621 (1973)

11. Hwang, J., Kim, J., Han, Y., Park, H.: An automatic cerebellum extraction method in T1-weighted brain MR images using an active contour model with a shape prior. Magnetic Resonance Imaging 29, 1014-1022 (2011)

12. Kim, H., Lepage, C., Evans, A.C., Barkovich, J., Xu, D.: NEOCIVET: Extraction of cortical surface and analysis of neonatal gyrification using a modified CIVET pipeline. In: MICCAI, Proceedings. pp. 571-579 (2015)

13. van der Lijn, F., de Bruijne, M., Hoogendam, Y.Y., Klein, S., Hameeteman, R., Breteler, M.M.B., Niessen, W.J.: Cerebellum segmentation in MRI using atlas registration and local multi-scale image descriptors. In: ISBI, Proceedings. pp. 221-224 (2009)

14. Machairas, V., Faessel, M., Cárdenas-Peña, D., Chabardes, T., Walter, T., Decencière, E.: Waterpixels. IEEE Transactions on Image Processing 24(11), 3707-3716 (2015) 
15. Mahapatra, D.: Skull stripping of neonatal brain MRI: Using prior shape information with graph cuts. Journal of Digital Imaging 25, 802-814 (2012)

16. Makris, N., Schlerf, J.E., Hodge, S.M., Haselgrove, C., Albaugh, M.D., Seidman, L.J., Rauch, S.L., Harris, G., Biederman, J., Caviness Jr, V.S., Kennedy, D.N., Schmahmann, J.D.: MRI-based surface-assisted parcellation of human cerebellar cortex: An anatomically specified method with estimate of reliability. NeuroImage 25, 1146-1160 (2005)

17. Makropoulos, A., Counsell, S.J., Rueckert, D.: A review on automatic fetal and neonatal brain MRI segmentation. NeuroImage 170, 231-248 (2017)

18. Makropoulos, A., Ledig, C., Aljabar, P., Serag, A., Hajnal, J.V., Edwards, A.D., Counsell, S.J., Rueckert, D.: Automatic tissue and structural segmentation of neonatal brain MRI using Expectation-Maximization. In: MICCAI NeoBrainS12, Proceedings. pp. 9-15 (2012)

19. Marlow, N., Wolke, D., Bracewell, M.A., Samara, M., Group, E.S.: Neurologic and developmental disability at six years of age after extremely preterm birth. The New England Journal of Medicine 352, 9-19 (2005)

20. Melbourne, A., Cardoso, M.J., Kendall, G.S., Robertson, N.J., Marlow, N., Ourselin, S.: NeoBrainS12 Challenge: Adaptive neonatal MRI brain segmentation with myelinated white matter class and automated extraction of ventricles I-IV. In: MICCAI NeoBrainS12, Proceedings. pp. 16-21 (2012)

21. Péporté, M., Ghita, D.E.I., Twomey, E., Whelan, P.F.: A hybrid approach to brain extraction from premature infant MRI. In: SCIA, Proceedings. pp. 719-730 (2011)

22. Prastawa, M., Gilmore, J.H., Lin, W., Gerig, G.: Automatic segmentation of MR images of the developing newborn brain. Medical Image Analysis 9, 457-466 (2005)

23. Randrianasoa, J.F., Kurtz, C., Desjardin, E., Passat, N.: Binary partition tree construction from multiple features for image segmentation. Pattern Recognition 84, 237-250 (2018)

24. Randrianasoa, J.F., Kurtz, C., Gançarski, P., Desjardin, E., Passat, N.: Evaluating the quality of binary partition trees based on uncertain semantic ground-truth for image segmentation. In: ICIP, Proceedings. pp. 3874-3878 (2017)

25. Randrianasoa, J.F., Kurtz, C., Gançarski, P., Desjardin, E., Passat, N.: Intrinsic quality analysis of binary partition trees. In: ICPRAI, Proceedings. pp. 114-119 (2018)

26. Romero, J.E., Coupé, P., Giraud, R., Ta, V.T., Fonov, V., Park, M.T.M., Chakravarty, M.M., Voineskos, A.N., Manjón, J.V.: CERES: A new cerebellum lobule segmentation method. NeuroImage 147, 916-924 (2017)

27. Rousseau, F., Oubel, E., Pontabry, J., Schweitzer, M., Studholme, C., Koob, M., Dietemann, J.L.: BTK: An open-source toolkit for fetal brain MR image processing. Computer Methods and Programs in Biomedicine 109, 65-73 (2013)

28. Saeed, N., Puri, B.: Cerebellum segmentation employing texture properties and knowledge based image processing: Applied to normal adult controls and patients. Magnetic Resonance Imaging 20, 425-429 (2002)

29. Salembier, P., Garrido, L.: Binary partition tree as an efficient representation for image processing, segmentation, and information retrieval. IEEE Transactions on Image Processing 9, 561-576 (2000)

30. Salembier, P., Wilkinson, M.H.F.: Connected operators: A review of region-based morphological image processing techniques. IEEE Signal Processing Magazine 26, 136-157 (2009)

31. Serag, A., Blesa, M., Moore, E.J., Pataky, R., Sparrow, S.A., Wilkinson, A.G., Macnaught, G., Semple, S.I., Boardman, J.P.: Accurate learning with few atlases 
(ALFA): An algorithm for MRI neonatal brain extraction and comparison with 11 publicly available methods. Scientific Reports 6, 23470 (2016)

32. Stoodley, C.J., Limperopoulos, C.: Structure-function relationships in the developing cerebellum: Evidence from early-life cerebellar injury and neurodevelopmental disorders. Seminars in Fetal and Neonatal Medicine 21, 356-364 (2016)

33. Tustison, N.J., Avants, B.B., Cook, P.A., Zheng, Y., Egan, A., Yushkevich, P.A., Gee, J.C.: N4ITK: Improved N3 bias correction. IEEE Transactions on Medical Imaging 29, 1310-1320 (2010)

34. Wang, L., Shi, F., Lin, W., Gilmore, J.H., Shen, D.: Automatic segmentation of neonatal images using convex optimization and coupled level sets. NeuroImage 58, 805-817 (2011)

35. Wang, L., Shi, F., Yap, P.T., Gilmore, J.H., Lin, W., Shen, D.: 4D multi-modality tissue segmentation of serial infant images. PLoS One 7, e44596 (2012)

36. Xue, H., Srinivasan, L., Jiang, S., Rutherford, M., Edwards, A.D., Rueckert, D., Hajnal, J.V.: Automatic segmentation and reconstruction of the cortex from neonatal MRI. NeuroImage 38, 461-477 (2007)

37. Yang, Z., Ye, C., Bogovic, J.A., Carass, A., Jedynak, B.M., Ying, S.H., Prince, J.L.: Automated cerebellar lobule segmentation with application to cerebellar structural analysis in cerebellar disease. NeuroImage 127, 435-444 (2016) 